www.jmscr.igmpublication.org

Impact Factor 5.84

Index Copernicus Value: 83.27

ISSN (e)-2347-176x ISSN (p) 2455-0450

crossref DOI: https://dx.doi.org/10.18535/jmscr/v5i4.199

Journal Of Medical Science And Clinical Research

\title{
Clinically detected Enteric Fever in Children - Blood Culture as a Diagnostic Aid
}

Authors

\section{Dr Kanai Lal Barik, Dr Rituparna Bhattacharya ${ }^{2}$, Dr Uttam Kumar Paul ${ }^{3 *}$, Dr Arup Bandyopadhyay ${ }^{4}$}

${ }^{1}$ Professor, Dept. of Pediatrics; Burdwan Medical College and Hospital, Burdwan, West Bengal

${ }^{2}$ Assistant Professor, Dept. of Microbiology; M.G.M Medical College and Hospital, Kishanganj, Bihar

Pin-855107

${ }^{3}$ Professor, Dept. of Medicine, MGM Medical College and Hospital, Kishanganj, Bihar Pin-855107

${ }^{4}$ Professor and Head, Dept. of Physiology, MGM Medical College and Hospital, Kishanganj Pin-855107

*Corresponding Author

Dr Uttam Kumar Paul*

Prayas, Dr. B. C. Roy Sarani, P.O - Raiganj, Dist. - Uttar Dinajpur, West Bengal, India Pin 733134

Email:druttam131065@gmail.com, Mobileno.:9434041237

\begin{abstract}
Enteric fever is considered a globally threatening disease in several developing countries, caused by the bacterium "Salmonella enterica serovar Typhi". Taking diagnosis into concern, blood culture technique is the most reliable diagnostic method, but is complicated and time consuming to be applicable in developing countries, especially in remote places and villages. In such unfavorable circumstances, we attempted to understand the current feasibility of blood culture as a diagnostic aid in clinically detected enteric fever patients in children population below 12 year age group to gain insight in order to develop better diagnostic strategies in the future. A total of 151 patients, of both sexes, below 12 years of age with fever of 3 or more days without any history of antibiotic therapy were included for the study. Blood sample was collected from each patient, and culture was carried out for salmonella using media, the results analyzed thereafter. The results showed that out of 151 clinically detected enteric fever patients, 43 (28\%) were positive for typhoid (S.typhi) and 19 (12\%) were positive for paratyphoid A (S.paratyphi A). 62 patients showed positive blood culture results, i.e. 40\% of the patients.

Thus the mass inaccurate diagnosis and empirical therapy practiced within the community is largely unacceptable. Proper control measures and better diagnostic strategies are warranted for the effective management of this medically and socially devastating malady.

Keywords: Enteric Fever, Diagnostic Aid, Blood Culture, Children.
\end{abstract}

\section{Introduction}

Enteric fever is considered a globally threatening disease in several developing countries of the South-East Asia, namely India, Pakistan and the Bangladesh ${ }^{1}$.It is an acute febrile illness caused by the bacterium "Salmonella enterica serovar Typhi",2. In India, the incidence of enteric fever amounts to 493.5 cases per 100,000 population per year. In Bangladesh, it is cited to be 18.7 per thousand population per year in pre-school 
children, while it is 2.1 per thousand per year in older people. The same, in Pakistan is reported as 451.7 cases per 100,000 population per year in children aged 2-15 years. Such high prevalence rate hints the need of further studies in order to explore the situation of enteric fever cases especially in developing countries. Africa shows less understanding of the disease. This is due to lack of appropriate infrastructure to conduct clinical or epidemiological studies compared to Asia. In a study by Crump et. al., only the crude incidence in Africa was reported, i.e. 50 cases per 100,000 population per year out of the total population being 820 million of the country ${ }^{3}$. However, the incidence was higher in another study by Buckle et.al., being that of 724.6 cases per 100,000 population per year ${ }^{4}$ One confounding factor in these studies is the presence of non-typhoidal Salmonella cases which mimics typhoid and which is progressively fatal and rising in some areas.

Taking diagnosis into concern, enteric fever raises confusion among the Afro-Asian countries. Blood culture technique is the most reliable diagnostic method, but is time consuming and expensive ${ }^{5}$. Detection of the pathogen in blood samples through polymerase chain reaction is probably the best and most reliable method. Next in line, is the bone marrow culture which is the second best in the aspect of its sensitivity. Recently, the third method that hit across several developing countries because its sensitivity is $100 \%$ is the typhoid-specific immunoglobulin A estimated by ELISA technique. The method uses blood sample and involves the amplification of signal by isolation and incubation of peripheral blood lymphocytes ${ }^{6}$. One important drawback is that, all the above mentioned methods being too expensive and tedious, it is complicated and time consuming to be applicable in developing countries, especially in remote places and villages. The Widal test has been markedly unreliable in such limited settings as well as the new generation serology tests like Typhidot and Tubex ${ }^{7,8,9}$.
A study on knowledge, attitude and various practices about typhoid of general practitioners in several under-developed sections of India and found noticeable poverty and lack of infrastructure in the diagnosis and management of the disease ${ }^{10}$. In another study, the authors have studied the acceptability and sensibility of blood culture as a diagnostic tool in clinically suspected adult typhoid cases ${ }^{11}$. In such unfavorable circumstances, we attempted to understand the current feasibility of blood culture as a diagnostic aid in clinically detected enteric fever patients in children population below 12 year age group to gain insight in order to develop better diagnostic strategies in the future.

\section{Materials and Methods}

A total of 151 patients (84 males and 67 females) with fever of 3 or more days duration were included in the study. The patients were in the age range of below 12 years. Most patients were from rural and semi-urban background. Patients who underwent anti-bacterial drug therapy for any ailment were excluded from the study.

About 4-5 ml of blood was withdrawn from each patient ( 5 years and above), using standard aseptic precautions. For patients of less than 5 years of age, the volume of blood collected was 2-3 ml. Each sample was inoculated into $40-45 \mathrm{ml}$ of Brain heart infusion (BHI) broth and incubated at $37^{\circ} \mathrm{C}$. Subcultures were done on MacConkey agar plates on days $1,2,3$ and 7 , which were then incubated at $37^{\circ} \mathrm{C}$ for $18-24$ hours. Non-lactose fermenting colonies, if found, on the subculture plates were further picked up and examined through microscopy by Gram staining of the smears. Standard biochemical tests were performed, including the test for motility, for identification of Salmonella species. 


\section{Results}

Table 1: Showing presence of S. typhi and S.paratyphi A in blood culture of fever patients clinically diagnosed as Enteric fever.

\begin{tabular}{|l|c|c|c|}
\hline Results & Number of pts & S. typhi detected & S. paratyphi A detected \\
\hline Male & $84(56 \%)$ & $26(17 \%)$ & $11(7 \%)$ \\
\hline Female & $67(44 \%)$ & $17(11 \%)$ & $8(5 \%)$ \\
\hline Total & 151 & $43(28 \%)$ & $19(12 \%)$ \\
\hline
\end{tabular}

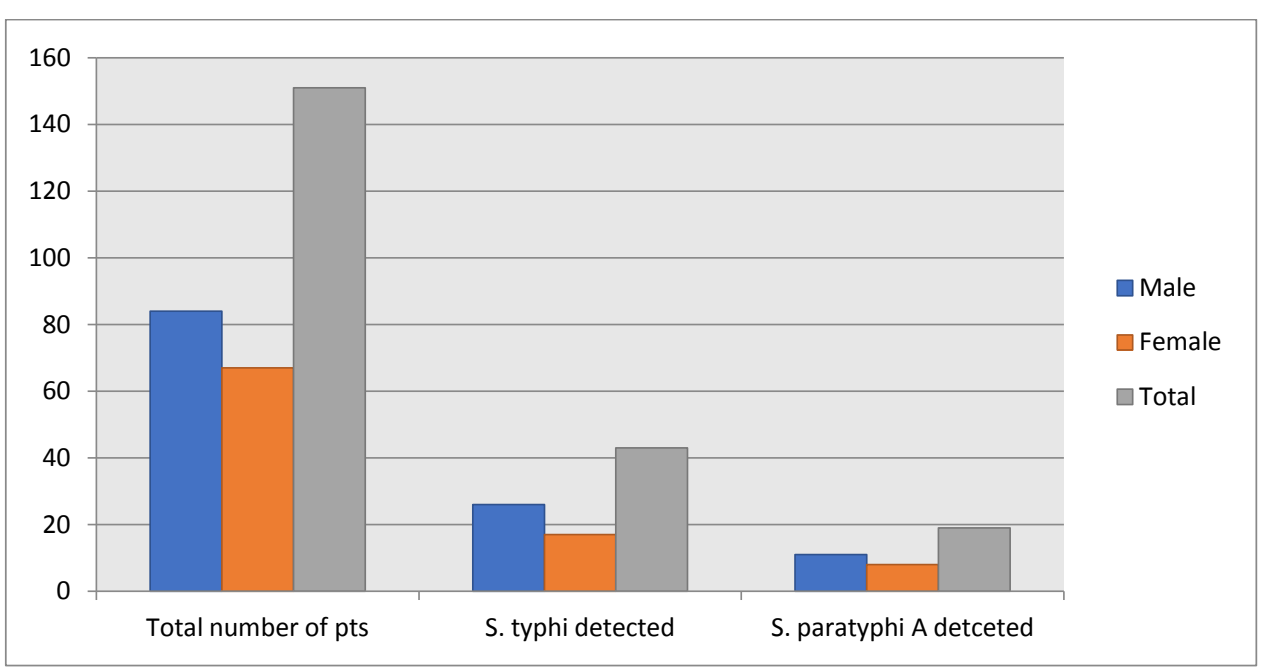

Column diagram: Depicting detection of of S. typhi and S.paratyphi A in blood culture of fever patients clinically diagnosed as Enteric fever.
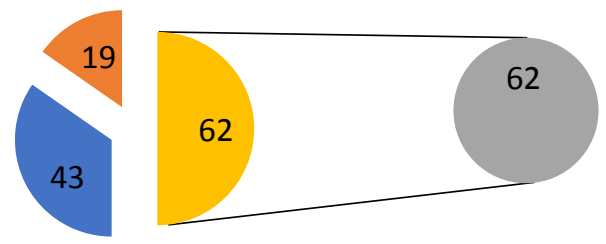

S. typhi detected
S. paratyphi A
detceted
Total

Pie diagram of S. typhi and S.paratyphi A in blood culture clinically diagnosed as Enteric fever.

Table 2: Duration of fever with positive blood culture.

\begin{tabular}{|lccc|}
\hline Duration of fever (days) & Number of patients & S.typhi isolated & S.paratyphi isolated \\
\hline $\mathbf{3}$ to $\mathbf{4}$ & 67 & 16 & 7 \\
\hline $\mathbf{5}$ to $\mathbf{6}$ & 53 & 15 & 7 \\
\hline $\mathbf{7}$ and more & 31 & 12 & 3 \\
\hline
\end{tabular}

\section{Discussion}

The study aims in determining the blood culture reports for the detection of S.typhi and S.paratyphi

A. Out of a total number of 151 children of below 12 years included for our study, 84 i.e. $56 \%$ were male and 67 i.e. $44 \%$ were female. S.typhi was isolated from blood culture of 26 male subjects, i.e. $17 \%$ of total study subjects and from 17 female subjects, i.e. $11 \%$ of total study subjects. S.paratyphi A was isolated from blood culture of 11 male subjects, i.e. $7 \%$ of total study subjects and from 8 female subjects, i.e. $5 \%$ of total study subjects. In our study, 67 patients presented with fever for 3 to 4 days of whom 16 were positive for S.typhi culture and 7 were positive for S.paratyphi A. Next, in total 53 patients presented with fever 
for 5 to 6 days, of whom 15 were positive for S.typhi culture and 7 were positive for S.paratyphi A culture. Again, 31 patients presented with fever for 7 days and more, of whom 12 were positive for S.typhi culture and 3 were positive for S.paratyphi A. In total, 43 (i.e. 28\%) subjects had positive culture for S.typhi and 19 (i.e. 12\%) subjects had positive culture for S.paratyphi A. This shows that, among the 151 subjects included in the study, 62 subjects showed positive blood culture for enteric fever which was $\sim 40 \%$ of the total study subjects.

Diagnosis of enteric fever is done in most countries primarily by blood culture, and also by stool or urine cultures and serological tests. In one study, most of the patients with enteric fever are children or young adults from 5 to 25 years of age $^{12}$. Another study showed that, among 273 blood cultures from clinically suspected enteric fever cases, S.typhi was detected in $7(2.6 \%)$ and S.paratyphi in $4(1.5 \%)$ of patients. The same study, using Widal test, also showed that TO antigen was positive (>1/80 dilution) in $47 \%$ of febrile patients, and $\mathrm{TH}$ antigen was positive in $26 \%$ cases (cutoff $>1 / 160$ ). Positive for both TO and $\mathrm{TH}$ were $24.4 \%{ }^{5}$. In a similar study, among the total of 67 adult patients clinically marked for typhoid fever, S.typhi was detected in only 8 (11.9\%) patients and S.paratyphi A was detected in only $3(4.5 \%)$ patients. In total, positive blood culture was detected in only some cases, i.e. 11 patients $(16.4 \%)^{11}$. A standard bacteriological laboratory is a definite prerequisite for blood culture at any site. One important criteria for proper blood culture is the immediate inoculation of the blood into appropriate media once withdrawn, thus maintaining proper temperature regulations of both. Moreover, the blood should be collected keeping all the aseptic precautions and which necessitates the requirement of a highly skilled and experienced technician. The volume of blood to be collected being variable poses another problem. Like, for certain adults and children, 10$15 \mathrm{ml}$ of blood is ideally collected, while for preschool children and toddlers, quantity of only 2-4 $\mathrm{ml}$ is sufficient. This might be because the samples of younger children yield more number of bacterial colonies than the adults ${ }^{11}$. It is unwise to compromise on the volume of blood since it readily reduces the sensitivity of the test. Difficulty in collection of small volumes of blood sometimes raises suspicion on the blood culture technique in diagnosis of enteric fever and therefore demands an alternate reliable and acceptable method to replace the former. Another concern is keeping the blood culture bottle aseptic for accurate diagnosis. Even a slight human error may lead to a false positive result or pseudobacteraemia. The same occurs when the exact recommended volume is not strictly maintained.

S.typhi infections are frequently reported from endemic regions due to poor hygiene conditions. Practices of open defecation and urination in the fields by infected persons spread infection. Thereafter, bacterial carriage via insects, namely flies and cockroaches to food products are an important source of disease transmission. Use of contaminated water for consumption is the prime cause of infection in developing countries, whereas in the developed countries it is food contaminated by droplet infection transmitted via healthy, asymptomatic carriers (viz. Mary Mallone - Typhoid Mary). Therefore improving the awareness about sanitary hygiene practices and household cleanliness especially in rural areas is the foremost step against prevention of enteric fever. Construction and use of sanitary latrines in every home and also on the roads and pavements should be the first idea to improve hygiene standards in this country.

Unfortunately, there seems to be very little scope of diagnostic and therapeutic success. Lack or no access to blood culture facility still prevails in many rural and semi-urban villages of our society, in spite of the fact that this test is the gold standard for the diagnosis of enteric fever.

We have, in our study, presented that out of 151 total patients clinically suspected to have enteric fever, 62 showed positive blood culture results, i.e. 40\% of the patients. Among them, 43 (28\%) 
were positive for typhoid (S.typhi) and 19 (12\%) were positive for S.paratyphi A. In fact, during clinical practice, these patients would have wrongly received therapy with antibiotics before reaching definitive diagnosis, thus leading to drug resistance and many obvert side effects.

Under such circumstantial pressure, and being a citizen of this society, it is our responsibility to come together, unify as members of the healthcare community and stand headstrong against these challenges in order to help our society to get proper diagnosis and treatment of enteric fever within time.

\section{Conclusion}

To conclude, it can be said that our study being a small community based one, it is clear that out of the total population included, only a small section $(\sim 40 \%)$ of the patients were clinically deemed to have enteric fever. Thus the mass inaccurate diagnosis and therapy prevalent in the community is largely unbecoming of scientific acceptance. Proper control measures should be devised and remedy sought for the hindrance faced during management of this under-diagnosed, medically under-reported and socially devastating malady.

\section{References}

1. Wain J, Hendriksen RS, Mikoleit ML, Keddy KH, Ochiai RL. Typhoid fever. The Lancet. 21 March, 2015; 385(9973): 11361145.

2. Christopher MP, Hien TT, Dougan G, Nickolas JW, Farrar JJ. Typhoid fever. NEJM 2002; Nov, 28; 347(22): 1770-1782.

3. Crump JA, Luby SP, Mintz ED. The global burden of typhoid fever. Bull World Health Organ 2004; 82: 346-53.

4. Buckle GC, Walker CL, Black RE. Typhoid fever and paratyphoid fever: systemic review to estimate global morbidity and mortality for 2010. J Glob Health 2012;2:10401.
5. Andualem G, Abebe T, Kebede N, GebreSelassie S, Mihret A, Alemayehu H. A comparative study of Widal test with blood culture in the diagnosis of typhoid fever in febrile patients. BMC Res Notes, 2014; 7: 653.

6. Khanam F, Sheikh A, Sayeed MA, et al. Evaluation of a typhoid/paratyphoid diagnostic assay (TP Test) detecting antisalmonella IgA in secretions of peripheral blood lymphocytes in patients in Dhaka, Bangladesh. Plos Negl Trop Dis 2013; 7: e2016.

7. Dutta S, Sur D, Manna B, et al. Evaluation of new-generation serologic tests for the diagnosis of typhoid fever: data from a community based surveillance in Calcutta, India. Diagn Microbiol Infect Dis 2006; 56: 359-65.

8. Ley B, Thriemer K, Ame SM, et al. Assessment and comparative analysis of a rapid diagnostic test (Tubex®) for the diagnosis of typhoid fever among hospitalized children in rural Tanzania. BMC Infect Dis. 2011; 11: 147.

9. Keddy KH, Sooka A, Letsoalo ME, et al. Sensitivity and specificity of typhoid fever rapid antibody tests for laboratory diagnosis at two sub-Saharan African sites. Bull World Health Organ. 2011; 89: 240-47.

10. Paul UK, Barik KL, Sinharay K, Banik S, Bandyopadhyay A. Knowledge, Attitude and Practice of General Practitioners Regarding Typhoid Fever. Int J Sci Stud. January 2016; 3(10): 1-4.

11. Bhattacharya P, Saha BK, Paul UK, Bandyopadhyay A. Blood Culture in Clinically Suspected Typhoid Fever. Int J Sci Stud. 2017; 4(11): 1-4.

12. Stuart BM, Pullen RL. Typhoid; clinical analysis of 360 cases. Arch Intern Med (Chic) 1946; 78: 629-61. 